\title{
ON KRULL'S CONJECTURE CONCERNING VALUATION RINGS
}

\author{
MASAYOSHT NAGATA
}

Introduction. Previously W. Krull conjectured ${ }^{1)}$ that every completely in tegrally closed primary ${ }^{2)}$ domain of integrity is a valuation ring. The main purpose of the present paper is to construct in $\S 1$ a counter example against this conjecture. In $\$ 2$ we show a necessary and sufficient condition that a field is a quotient field of a suitable completely integrally closed primary domain of integrity which is not a valuation ring.

By a ring we mean a commutative ring with identity. We refer to the notations like $D_{n}$ as the ring of quotients of $p$ with respect to $D$ when $n$ is a ring and $p$ is a prime ideal of $D$.

\section{A counter example.}

Let $K$ be an algebraically closed field with a non-trivial special valuation $w$ whose value group $G$ does not fill up all real numbers. Take a positive number $\alpha$ which is not in $G$. Consider a rational function field $K(x)$ of one variable $x$ with constant field $K$. Let us define the following two types of valuations of $K(x)$ which are extensions of $w$ : (1) For every element $e$ of $K$ such that $\alpha<w(e)<2 \alpha,{ }^{3)}$ we define a valuation $w_{e}$ (of $K(x)$ ) such that

$$
w_{0}\left(\sum_{i=0}^{n} a_{i}(x+e)^{i}\right)=\min \left(w\left(a_{i}\right)+2 \alpha i\right) \quad\left(a_{i} \in K\right) .^{4)}
$$

(2) For every real number $\lambda$ such that $\alpha \leqq \lambda \leqq 2 \alpha$, we define a valuation $w_{\lambda}$ such that

$$
w_{\lambda}\left(\sum_{i=0}^{n} a_{i} x^{i}\right)=\min \left(w\left(a_{i}\right)+\lambda i\right) \quad\left(a_{i} \in K\right) .
$$

THEOREM 1. Let $\mathrm{n}_{e}$ and $\mathrm{n}_{\lambda}$ be the valuation rings determined by $w_{e}$ snd $w_{\lambda}$ respectively $(\alpha<w(e)<2 \alpha, \alpha \leqq \lambda \leqq 2 \alpha)$ and let o be the intersection of all such $\mathfrak{I}_{e}$ and $\mathrm{D}_{\lambda}$. Then $\mathfrak{D}$ is completely integrally closed and primary, but $\mathrm{o}$ is not a

Received May 22, 1951.

J) W. Krull, Beiträge zur Arithmetik kommutativer Integritätsbereiche II, Math. Zeit. 41 (1936). p, 670.

$\Rightarrow$ A ring is called primary if it has at most one proper prime ideal.

3) Observe the fact that $2 \alpha \notin G$, because $K$ is algebraically closed.

4) Since $2 x \notin G, w_{e}$ is uniquely determined by the relation $w_{e}(x+e)=2 x$. 
valuation ring.

Proof. Let $c(\neq 0)$ be an element of 0 . First we prove that (1) if $w_{\lambda_{\theta}}(c)$ $=0$ for some $\lambda_{0}\left(\alpha \leqq \lambda_{0} \leqq 2 \alpha\right)$, then $w_{\lambda}(c)=0$ and $w_{e}(c)=0$ for every $w_{\lambda}$ and $w_{e}$, and that (2) if $w_{\alpha}(c)>0$, there exist the least and the largest values $\varepsilon>0$ and $\delta$ among values of $c$ taken by $w_{\lambda}$ and $w_{e}(\alpha \leqq \lambda \leqq 2 \alpha, \alpha<w(e)<2 \alpha)$.

Since $K$ is algebraically closed, $c$ is of the form

$$
c_{0} \prod_{i=1}^{n}\left(x+a_{i}\right) / \prod_{j=1}^{m}\left(x+b_{j}\right) \quad\left(c_{0}, a_{i}, b_{j} \in K\right) .
$$

Every factor $x+d\left(d=a_{i}\right.$ or $\left.b_{j}\right)$ such that $w(d)>2 \alpha$ may be replaced by $x$, since we only consider the values of $c$ taken by $w_{\lambda}$ and $w_{e}$. Similarly we may replace by $d$ every factor $x+d\left(d=a_{i}\right.$ or $\left.b_{j}\right)$ such that $w(d)<\alpha$. Therefore we may assume without loss of generality that (i) $\alpha<w\left(a_{i}\right)<2 \alpha$ or $a_{i}=0$, $\alpha<w\left(b_{j}\right)<2 \alpha$ or $b_{j}=0$ for each $i$ and $j(1 \leqq i \leqq n, 1 \leqq j \leqq m)$, (ii) $a_{i} \neq b_{j}$ for every pair $(i, j)$ and (iii) $w\left(a_{i}\right) \leqq w\left(a_{i+1}\right), w\left(b_{j}\right) \leqq w\left(b_{j+1}\right)(1 \leqq i<n, 1 \leqq j<m)$.

First we assume that $w_{\lambda_{0}}(c)=0$ for some $\lambda_{0}\left(\alpha \leqq \lambda_{0} \leqq 2 \alpha\right)$. If there exists one $j_{1}$ such that $w\left(b_{j_{1}}\right)=\lambda_{0}$, then we have $w_{b_{j_{1}}}(c)<0$, which is a contradiction. Therefore no $w\left(b_{j}\right)$ is equal to $\lambda_{0}$. Assume that $w\left(a_{i}\right)<\lambda_{0}$ if $i \leqq i_{0}, w\left(a_{i}\right)=\lambda_{0}$ if $i_{j}<i \leqq i_{0}+s, w\left(a_{i}\right)>\lambda_{0}$ if $i>i_{0}+s ; w\left(b_{j}\right)<\lambda_{0}$ if $j \leqq j_{0}, w\left(b_{j}\right)>\lambda_{0}$ if $j>j_{0}$. Set $\lambda_{1}=\max \left(\alpha, w\left(a_{i_{0}}\right), w\left(b_{j_{0}}\right)\right), \lambda_{2}=\min \left(2 \alpha, w\left(a_{i_{0}+s+1}\right), w\left(b_{j_{0}+1}\right)\right.$.

Then

$$
\begin{aligned}
& w_{\lambda_{1}}(c)=w\left(c_{0}\right)+\sum_{i \leqq i_{0}} w\left(a_{i}\right)-\sum_{j \leqq j_{0}} w\left(b_{j}\right)+\left(n-i_{0}\right) \lambda_{1}-\left(m-j_{0}\right) \lambda_{1} \geqslant 0, \\
& w_{\lambda_{0}}(c)=w\left(c_{0}\right)+\sum_{i \leqq i_{0}} w\left(a_{i}\right)-\sum_{j \leqq j_{0}} w\left(b_{j}\right)+\left(n-i_{0}\right) \lambda_{c}-\left(m-j_{0}\right) \lambda_{0}=0, \\
& w_{\lambda_{2}}(c)=w\left(c_{0}\right)+\sum_{i \geqq i_{0}} w\left(a_{i}\right)-\sum_{j \leqq j_{0}} w\left(b_{j}\right)+s \lambda_{0}+\left(n-i_{0}-s\right) \lambda_{2}-\left(m-j_{0}\right) \lambda_{2} \approx 0 .
\end{aligned}
$$

Hence we have

$$
w_{\lambda_{1}}(c)=w_{\lambda_{1}}(c)-w_{\lambda_{0}}(c)=\left(n-i_{0}\right)\left(\lambda_{1}-\lambda_{0}\right)-\left(m-j_{0}\right)\left(\lambda_{1}-\lambda_{0}\right) \geqslant 0,
$$

whence $n-i_{0} \leqq m-j_{0}{ }^{5}$

Similarly we have

$$
w_{\lambda_{2}}(c)=w_{\lambda_{2}}(c)-w_{\lambda_{0}}(c)=\left(n-i_{0}-s\right)\left(\lambda_{2}-\lambda_{0}\right)-\left(m-j_{0}\right)\left(\lambda_{2}-\lambda_{0}\right) \geqq 0,
$$

whence $n-i_{0}-s \gtrsim m-j_{0}$.

Thus we have $s=0$ and $n-i_{0}=m-j_{0} . \quad s=0$ shows that no $w\left(a_{i}\right)$ is equal to $\lambda_{c}$. Further, $n-i_{0}=m-j_{0}, s=0$ show $w_{\lambda_{1}}(c)=w_{\lambda_{2}}(c)=0$. Therefore neither $w\left(a_{i}\right)$ nor $w\left(b_{j}\right)$ are equal to $\lambda_{1}$ or $\lambda_{2}$, by the above observation. This means that $\lambda_{1}=\alpha$ and $\lambda_{2}=2 \alpha$. From $\lambda_{1}=\alpha$ we have that $i_{0}=j_{0}=0$, whence $m=n$; From $\lambda_{2}=2 \alpha$ we have that $a_{i}=0, b_{j}=0(1 \leqq i \leqq n, 1 \leqq j \leqq m)$. By our assumption

5) If $\alpha=\lambda_{0}$ or $2 \alpha=\lambda_{0}$, we see easily that $n-i_{0}=m-j_{0}$ because $\alpha \neq G$. In this case, $s=0$ is also clear. 
that $a_{i} \neq b_{j}$, it follows that $m=n=0$, i.e., $c=c_{0} \in K$. Since $w_{\lambda_{0}}(c)=0$, we have $w(c)=0$. This proves (1). Next assume that $w_{\alpha}(c)>0$. Let us consider $w_{\lambda}(c)$ as a function of variable $\lambda(\alpha \leqq \lambda \leqq 2 \alpha)$. Then it is evidently continuous, and it takes the least and the largest values $\varepsilon_{1}$ and $\delta_{1}$ in $\alpha \leqq \lambda \leqq 2 \alpha$. By virtue of (1), we see that $\varepsilon_{1}$ is positive. Then (2) follows easily from the fact that $w_{e}(c)$ $\neq w_{w(e)}(c)$ holds only if $e$ is one of $a_{i}$ or $b_{j}$ and in this case $w_{e}(c) \notin G$, whence $w_{e}(c) \neq 0$.

These being proved, we see that 0 is primary. Let $a(\neq 0)$ and $b(\neq 0)$ be two non-units in $\mathrm{n}$. Then there exist positive numbers $\varepsilon$ and $\delta$ such that $w_{\lambda}(a)$ $\geqslant \varepsilon, w_{e}(a) \geqq \varepsilon, w_{\lambda}(b) \leqq \delta, w_{e}(b) \leqq \delta(\alpha \leqq \lambda \leqq 2 \alpha, \alpha<w(e)<2 \alpha)$. Let $k$ be an integer such that $k_{\varepsilon}>\delta$. Then we have $w_{\lambda}\left(a^{k} / b\right) \geqslant 0, w_{e}\left(a^{k} / b\right) \geqslant 0(\alpha \leqq \lambda \leqq 2 \alpha, \alpha<w(e)$ $<2 \alpha)$, whence $a^{k} / b \in \mathrm{D}$, i.e., $a^{k} \in b$.

It is evident that $\mathfrak{D}$ is completely integrary closed, because $\mathfrak{D}$ is an intersection of special valuation rings. That $\mathfrak{b}$ is not a valuation ring follows from that $e / x \notin 0, x / e \notin 0$ if $\alpha<w(e)<2 \alpha$.

\section{An existence theorem.}

LEMMA 1 . Let $\mathfrak{r}$ be an integrally closed integral domain which has only one maximal ideal $p_{0}$. Let $K$ be the quotient field of $r$. If $Z$ is a field containing $K, \mathfrak{p}_{\mathfrak{p}} \cap K=\mathfrak{r}$, where $\mathfrak{b}$ is the totality of $\mathfrak{r}$-integers in $Z$ and $\mathfrak{p}$ a maximal ideal of $\mathfrak{D}$.

Proof. We may assume without loss of generality that $Z$ is algebraic over $K$ because the quotient field of $\mathfrak{o}$ is algebraic over $K$.

First we assume that $Z$ is finite normal over $K$. Let $\left\{\sigma_{1}, \ldots, \sigma_{n}\right\}$ be the totality of automorphisms of $Z$ over $K$. We show that every maximal ideal of $\mathfrak{b}$ is one of $p^{\circ i}$ : Assume that a maximal ideal $\mathfrak{q}$ of $\mathfrak{b}$ is none of $\mathfrak{p}^{\circ i}$. Then there exists an element $c$ of $\mathfrak{q}$ such that $c \notin p^{\sigma_{i}}$ for every $i=1, \ldots, h$. A power $e$ of $\prod_{i=1}^{h} c^{\sigma_{i}}$ is in $K$, whence in $\mathfrak{r}$. Since $c \in \mathfrak{q}$, we have $e \in p_{n}$, whence $e \in \mathfrak{p}^{6)}$ Therefore one of $c^{\sigma_{i}}$ must be in $\mathfrak{p}$, i.e., $c$ is in some $p^{\sigma_{i}}$, which is a contradiction. This being shown, we have $\mathfrak{D}=\bigcap_{i=1}^{h}\left(\mathfrak{a}_{\mathfrak{p}}\right)^{\sigma_{i}}$. Therefore $\mathfrak{o}_{\mathfrak{p}} \cap K=\left(\mathfrak{o}_{\mathfrak{p}}\right)^{\sigma_{i}} \cap K=\left(\bigcap_{i=1}^{h}\left(\mathfrak{o}_{\mathfrak{p}}\right)^{\sigma_{i}}\right)$ $\cap K=\mathfrak{o} \cap K=\mathrm{r}$.

Next we assume that $Z$ is finite algebraic over $K$. Let $Z^{*}$ be a field containing $Z$ which is finite normal over $K$. Let $D^{*}$ be the totality of $\mathfrak{r}$-integers in $Z^{*}$ and let $\mathfrak{p}^{*}$ be a maximal ideal of $\mathfrak{D}^{*}$ which contains $\mathfrak{p}^{*}$. Then evidently $\mathfrak{o}_{\mathfrak{p}^{*}}^{*} \supseteq \mathfrak{p}_{\mathfrak{p}}$. Since $\mathfrak{o}_{\mathfrak{p}^{*}}^{*} \cap K=\mathfrak{r}$, we have $\mathfrak{o}_{\mathfrak{p}} \cap K=\mathfrak{r}$.

Making use of this, we prove the general case. Let $c$ be an element of $\mathrm{B}_{\mathfrak{p}} \cap K . \quad c$ may be written in a form $a / b(a, b \in \mathrm{n}, b \neq \mathfrak{p})$. We consider $Z^{*}=K(a$, $b)$. We set $\mathfrak{D}^{*}=\mathfrak{o} \cap Z^{*}$, and $\mathfrak{p}^{*}=\mathfrak{p} \cap \mathfrak{o}^{*}$. Then $\mathfrak{p}^{*}$ is a maximal ideal because $\mathfrak{D}$

6) Because $\mathfrak{b}$ is integral over $r, f 0=x \cap p=x \cap q$. 
is integral over $0^{*}$. It is clear that $a, b \in 0^{*}, b \notin p^{*}$ whence $0_{p^{*}}^{*} \exists c$. Since $Z^{*}$ is finite over $K$, we have $\mathrm{o}_{p^{*}}^{*} \cap K=\mathrm{r} \exists c$, which proves our assertion.

Lemma 2. Let $K$ be a field with a valuation ring $\mathfrak{v}$ and let $Z$ be a field containing $K$ which is algebraic over $K$. Let $\mathfrak{D}$ be the totality of $\mathfrak{v}$-integers in $Z$ and let $\left\{p_{\lambda} ; \lambda \in A\right\}$ be the totality of maximal ideals of 0 . Then every valuation ring $\mathfrak{w}$ of $Z$, such that the valuation given by $\mathfrak{w}$ is an extension of that given by $\mathfrak{v}$, is one of $\mathfrak{p}_{p_{\lambda}}(\lambda \in A)$. Conversely, every $\mathfrak{p}_{p_{\lambda}}(\lambda \in A)$ is a valuation ring.

Proof. It is clear that any such valuation ring $\mathfrak{w}$ contains one of $\mathfrak{o}_{\mathfrak{p}_{\lambda}}$. Hence we have only to prove the converse part. But this follows immediately from the following facts :

$(1)^{7)}$ An integrally closed domain $m$ of integrity is a multiplication ring if and only if $m_{\mathfrak{p}}$ is a valuation ring for every maximal ideal $p$ of $m$.

$(2)^{8)}$ Let $\mathfrak{m}$ be a multiplication ring with quotient field $K$. If a field $Z$ containing $K$ is algebraic over $K$, then the totality $n$ of $m$-integers in $Z$ is also a multiplication ring and $Z$ is the quotient field of $r$.

Lemma 3. Let $\mathfrak{r}$ be a completely integrally closed integral domain with quotient field $K$. If $Z$ is a field containing $K$, the totality 0 of $r$-integers in $Z$ is also cmpletely integrally closed.

Proof. Assume that $Z$ is finite normal (algebraic) over $K$. Let $\left\{\sigma_{1}, \ldots\right.$, $\left.\sigma_{n}\right\}$ be the totality of automorphisms of $Z$ over $K$. Set $r=[Z: K] / h$. Assume that $(a / b)^{n} c \in \mathcal{0}$ for every natural number $n$, where $a, b$ and $c$ are non-zero elements of $s$. Let $f$ be an arbitrary elementary symmetric formula of $\left[(a / b)^{a_{1}}\right]^{r}$, $\ldots,\left[(a / b)^{\sigma_{n}}\right]^{r}$, and set $c^{\prime}=\left(\prod_{i=1}^{h} c^{\sigma_{i}}\right)^{r}$. Then $f^{n} c^{\prime} \in \mathrm{r}$, whence $f^{n} c^{\prime} \in \mathfrak{r}$ for every natural number $n$. This shows that $f \in \mathfrak{r}$, whence $a / b$ satisfies a monic equation with coefficient in $r$, i.e., $a / b \in \mathfrak{0}$, which proves our assertion when $Z$ is finite normal over $K$. This being proved, we can reduce our problem to the ganeral case by the same way as in the proof of Lemma 1 .

Theorem 2. Let $K$ be a field. Then there exists a completely integrally closed primary domain of integrity which is not a valuation ring such that its quotient field is $K$ if and only if $K$ satisfies one of the following two conditions:

(1) $K$ is of characteristic 0 and $K$ is not algebraic over its prime field.

(2) $K$ is of characteristic $p(\neq 0)$ and $K$ contains at least two algebraically independent elements over its prime field.

7) W. Krull, Beiträge zur Arithmetik kommutativer Integritätsbereiche, Math. Zeit. 41 (1936), Theorem 7 (p. 554).

8) Prüfer, Untersuchungen über die Teilbarkeitseigenschaften in Körpern, Crelle 168, p. 31 or 1. c. note 6) Theorem 8 (p. 555). 
Proof. (I) The case where $K$ satisfies neither of these conditions. Let 0 be any integrally closed ${ }^{9)}$ primary domain of integrity with quotient field $K$. When $K$ is algebraic over its prime field, let $K_{0}$ be its prime field. When $K$ is not algebraic over its prime field, let $K_{0}$ be its subfield which is isomorphic to the rational function field of one variable with its prime field as the constant field. Then evidently $0 \cap K_{0}$ is a valuation ring. Then by Lemma 2 it follows that $\mathrm{D}$ is also a valuation ring.

(II) Assume that $K$ satisfies one of the above two conditions. Then it is easy to see that there exists a subfield $K_{0}$ of $K$ such that $K_{0}$ has a non-trivial discrete special valuation and such that $K$ has transcendental degree 1 over $K_{0}$, that is, there exists an element $x$ of $K$ such that $x$ is not algebraic over $K_{0}$ and $K$ is algebraic over $K_{0}(x)$. Let $\bar{K}_{0}$ and $\bar{K}$ be the algebraic closures of $K_{0}$ and $K$ respectively. Then by Theorem 1 we can construct a completely integrally closed primary domain $x$ of integrity which is not a valuation ring and whose quotient field is $\bar{K}_{0}(x)$. Let $\bar{D}$ be the totality of $r$-integers in $\bar{K}$ and let $\bar{p}$ be a maximal ideal of $\bar{b}$. Set $n=\overline{p_{p}} \cap K$. Then since $r$ is completely integrally closed, $\bar{D}$ is so too by Lemma 3. Therefore $D$ is also completely integrally closed. Since $\mathfrak{r}$ is primary, so is $\overline{p_{p}}$ too, whence $\mathfrak{b}$ is primary. On the other hand, since $\overline{D_{p}}$ $\cap K_{1}(x)=\mathrm{r}$ by Lemma $1, \bar{p}$ is not a valuation ring and therefore $\mathfrak{o}$ is not a valuation ring again by virtue of Lemma 2, Thus our proof is complete.

Mathematical Institute, Nagoya University

9) We need not assume here that 0 is "completely" integrally closed. 